\title{
NUCLEAR AEROSOL BEHAVIOR DURING REACTOR ACCIDENTS
}

\author{
M. M. R. Williams \\ Nuclear Engineering Department, The University of Michigan, Ann Arbor, MI, 48109, U.S.A.
}

\begin{abstract}
Some early reactor accidents are recalled together with their associated environmental consequences. One such consequence is the generation of radioactive aerosol. We describe the various physical processes that such an aerosol cloud undergoes within the secondary containment building. These physical processes are then brought together quantitatively in a balance equation for the aerosol size spectrum as a function of position and time. Methods for solving this equation are discussed and illustrated by the method of moments based upon log-normal and modified gamma distributions. Current problems are outlined and directions for future work into aerosol behavior are suggested.
\end{abstract}

\section{INTRODUCTION}

The purpose of this article is to give an overview of the general problems confronting nuclear engineers when faced with an aerosol release within the containment vessel of a nuclear reactor. By an aerosol, we mean a suspension of fine particles in the atmosphere which can arise due to a core melt, leading to vaporized fission products or as the result of a fire. The typical size of such aerosol particles varies initially between 0.1 and 10 microns and we wish to know how they settle and disperse within the containment.

The way in which the importance of nuclear safety analysis has grown over the years is briefly reviewed, with particular emphasis on particulate (i.e. aerosol) release and the ways in which this is transported from place to place within the secondary containment building. We will then go on to discuss some more detailed mathematical and physical matters.

The amount of radionuclides released to the environment from a given reactor accident sequence has been called the 'source term' and knowledge of this is basic for any calculation of the subsequent dispersal of radioactive material. This is why it is so vital to understand aerosol behavior, which will enable containment buildings to be designed more effectively, with appropriately engineered safeguards (Wilson et al., 1985).

The development of safety assessments and procedures has by and large been spurred on by actual accidents that have taken place. The first serious such accident was that at the Windscale, air-cooled, graphite moderated reactor in 1957 which, due to a Wigner energy release, caught fire. This led to substantial amounts of radioactive material being deposited over
England, Wales and Northern Europe. It is estimated that about $10^{15} \mathrm{~Bq}$ of the iodine and about $10^{14} \mathrm{~Bq}$ of the caesium must have escaped. Since the reactor was not enclosed by a containment building the question of the effectiveness of such a structure could not be assessed; although there is no doubt that the filters in the stack proved very useful.

Another accident which strongly influenced subsequent thinking on the consequences to the public occurred in the SL-1 reactor in Idaho, U.S.A. in 1961. This was a $3 \mathrm{MW}$ BWR. Due to unauthorized removal of a control rod, there was a severe power excursion and steam explosion that resulted in damage and vaporization to about $20 \%$ of the core. Between 5 and $15 \%$ of the fission product inventory escaped from the reactor vessel, although less than $0.5 \%$ of iodine 131 was found outside the reactor building in the surrounding desert. It is believed, however, that this was more by luck than judgement because the reactor building was not specifically designed to contain radionuclides. It simply meant that because the reactor was not pressurized, there was no great force available to drive the radionuclide release.

These accidents, together with a generally more enlightened policy towards safety analysis, led to the idea of the maximum credible accident or as it is sometimes called the design basis accident. During the 1960s many new rules and regulations were laid down by national nuclear safety bodies regarding releases from various types of reactor and the concept of engineered safeguards such as emergency core cooling systems and containment sprays received much more serious consideration.

Undoubtedly the most significant milestone in reactor safety occurred in 1975 when Norman Rasmussen 
and his colleagues produced the reactor safety study, referred to by the report number Wash 1400 . This report was based on the premise that nuclear technology had reached a point where it should be possible to make realistic estimates of the probabilities and consequences of accidents that might proceed beyond the design basis accident and lead to core melt. Each accident sequence was assigned a probability, the value of which was obtained from historical data or engineering judgement. Whilst Wash 1400 had many limitations and shortcomings, it was the precursor of modern accident analysis based on probabilistic risk assessment, which was developed so effectively by Farmer (1967).

Ironically, it was not until after the accident at Three Mile Island that calculations showed that that particular accident (i.e. loss of feedwater transient) on a Babcock and Wilcox reactor had a probability of 1 in 300 years. Since Babcock and Wilcox reactors had an operating history of about 30 years the chance of an accident was by no means unlikely. This realization led to a general acceptance of probabilistic risk assessment as an important part of design and accident prevention.

A number of questions arose out of the TMI accident concerning the conservatism of the magnitude of the source term used. It was recognized, for example, that far less radioactive material was released from the reactor and its containment than might have been indicated by prevailing assessment methods. For example, much of the iodine was converted to caesium iodide, a highly water soluble and non-volatile substance. Thus the caesium iodide formed at TMI was largely retained within the containment structure in the water that remained in the primary system of the reactor.

As a result of these observations, and others, the technical community now believe that a more realistic evaluation of severe accident source terms is necessary. This aspect now forms a part of a greatly expanding programme of nuclear safety, supported internationally, on experimental and theoretical problems associated with physical and chemical phenomena in radionuclide release.

\section{AEROSOL BEHAVIOR}

There are, of course, many aspects of reactor accident analysis. Some are concerned with preventing accidents or at least minimizing the likelihood of their occurrence. Others are concerned with dealing with and mitigating the consequences of an accident when it has happened.

One particular area in this latter category which has been studied for some years is related to aerosol release.
We know, for example, that in a serious accident which involves core melt, fission product aerosols can be released into the containment vessel and if this fails, into the environment. In fact, even if the containment does not fail, there will always be some leakage to the environment through structural defects and diffusion. However, considerable interest has been shown in the calculation of the transport of aerosols within the various chambers of the containment.

This poses a very difficult problem for several reasons, two of which are as follows:

(1) The nature of the release is unknown so that the problem has to be understood for a wide range of input data.

(2) The geometrical structure through which the aerosol moves is uncertain if it is assumed that the accident itself has destroyed some of the structure.

We need, therefore, some quantitative way to describe the aerosol migration. This is done by the introduction of a distribution function $n(v, \mathbf{r}, t)$, where $n(v, \mathbf{r}, t) \mathrm{d} v \mathrm{~d} \mathbf{r}$ is the number of aerosol particles with volumes in the size range $v$ to $v+\mathrm{d} v$ in the volume element of space $\mathrm{dr}$ at $\mathbf{r}$ at time $t$. This function is the solution of a complex integro-differential equation. The equation can be written down but it contains many parameters that are themselves subject to uncertainty or have to be derived from other equations possibly linked to the aerosol equation. Even if approximate forms are used for these input parameters, the subsequent numerical solution of the equation in the complex geometry of the reactor is a formidable task.

A good pictorial description of the processes which are operating is given in the user's manual of the code (see Fig. 1). This is a code for severe accident analysis developed by Sandia National Laboratories for the USNRC.

There are three main areas of concern; (1) aerosols, (2) fission products and (3) thermal hydraulics.

Fission products cause heating and are converted to aerosols which are then transported by the thermal hydraulics.

In this paper we will discuss some approaches to one of these problems (that is, aerosol distribution) wherein it is assumed that the accident scenario has been defined so that leak paths, flow and thermal conditions and the internal source term are prescribed. A typical geometrical situation is shown in Fig. 2 where a PWR has been divided into six compartments. These compartments communicate with each other by gas, liquid and heat flow through the various flow paths. The environment could be added as another compartment if the leakage rates from the outer compartments are known. This is the type of geometry that one generally meets, although 


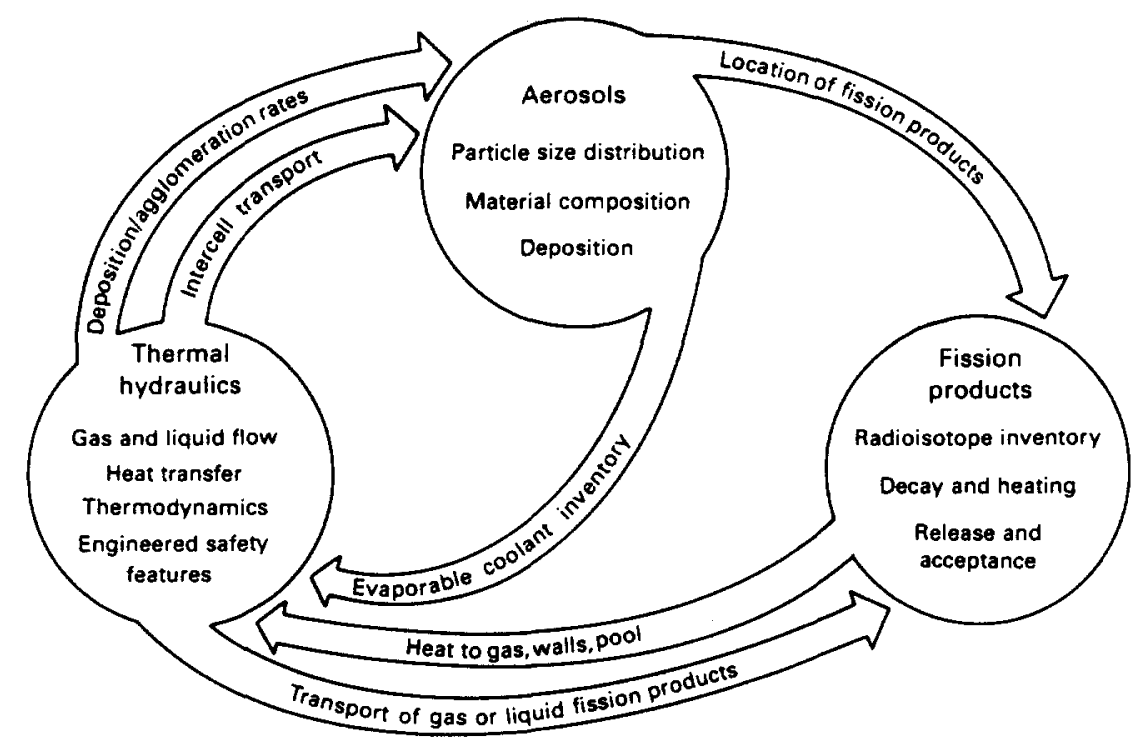

Fig. 1. The three basic phenomenological areas and their intercoupling. (From User's manual of the code CONTAIN 1.0 Sandia National Laboratories.)

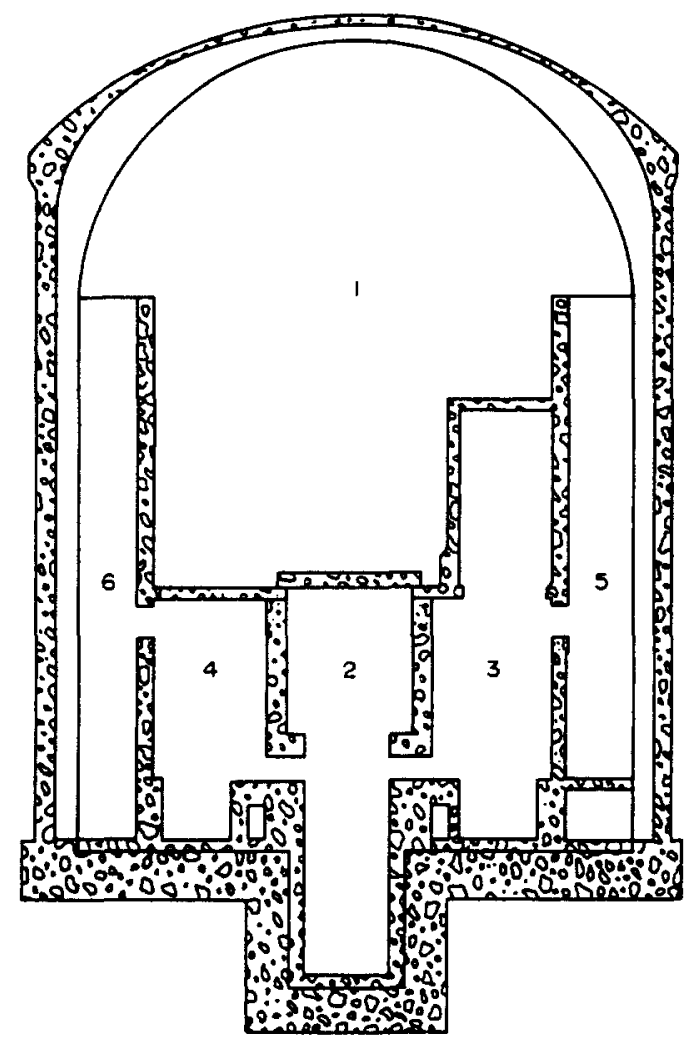

Fig. 2. Reactor containment building reduced to a configuration of six inter-connected compartments. (From User's manual of the code CONTAIN 1.0 Sandia National Laboratories.) clearly in a severe accident there may be structural damage. It is necessary to model the aerosol behavior in such a geometry.

\section{THE AEROSOL BALANCE EQUATION}

The aerosol distribution function should take into account the fact that there are different species of aerosol in the system, e.g. CsI, $\mathrm{CsOH}$, tellurium, core and concrete materials. This is particularly important when the source term is time dependent or when sources of different species have widely differing particle sizes. However, for simplicity, it will be assumed that we have only a single type of material present. Thus we need an equation for the particle distribution function $n(v, r, t)$ which takes the form (Friedlander, 1977):

$$
\begin{aligned}
& \frac{\partial n(v \mathbf{r}, t)}{\hat{c} t}+\nabla \cdot[\mathbf{U}(v, \mathbf{r}, t) n(v, \mathbf{r}, t)] \\
& +\nabla \cdot D(v, \mathbf{r}, t) \nabla n(v, \mathbf{r}, t)+\frac{\hat{c}}{\hat{c} v}[I(v, \mathbf{r}, t) n(v, \mathbf{r}, t)] \\
& \quad=\frac{1}{2} \int_{0}^{v} \mathrm{~d} u K(u, v-u ; \mathbf{r}, t) n(u, \mathbf{r}, t) n(v-u, \mathbf{r}, t) \\
& -n(v, \mathbf{r}, t) \int_{0}^{\infty} \mathrm{d} u K(u, v ; \mathbf{r}, t) n(u, \mathbf{r}, t)+Q(v, \mathbf{r}, t) .
\end{aligned}
$$

The physical situation corresponds to a burst of particles which settle, diffuse and coagulate and the equation tells us how the size spectrum changes with position and time. 
In this equation, $\mathbf{U}$ is the vector sum of the fluid and particle velocities and $D$ is the diffusion coefficient. Both quantities depend on the total volume of the particles. $I(t, \mathbf{r}, t)$ is the rate of increase or decrease of particle volume due to condensation or evaporation. The coagulation kernel $K(u, v ; \mathbf{r}, t)$ is a measure of the agglommeration rate of a particle of volume $u$ with a particle of volume $t$.

Finally, $Q(\ldots)$ is a source term which arises from the fission product release but could also be due to homogeneous nucleation. Also we note that due to the thermohydraulic behavior, the parameters, $U, D, I, K$ and $Q$ will depend on position and time.

The equation itself describes a balance for the particle distribution function $n(v, \mathbf{r}, t)$.

\subsection{Solution of the equation}

In order to solve the equation for a practical situation, it will be necessary to know the particle and fluid velocities, thus the equations of fluid flow must be solved, which involves detailed thermohydraulic calculations: At the same time these equations have to be coupled to the particles which, in this case at least, can be treated as non-interacting due to their low volume fraction. We also need the growth law for evaporation and condensation which clearly depends on the supersaturation of the surrounding vapor and its temperature distribution.

The diffusion coefficient arises from Brownian diffusion and can be calculated fairly well using standard methods.

The coagulation kernel $K(\ldots)$ is a vital part of the calculation and in order to illustrate the problems that it poses let us examine some of the more common mechanisms for coagulation.

3.1.1. Brownian motion. According to the diffusion equation for particles that are large compared to a mean free path, the value of $K$ for coagulation of particles of volumes $u$ and $v$ (radii $r_{1}$ and $r_{2}$ ) is;

$$
K_{\mathrm{B}}(u, v)=4 \pi\left(D_{1}+D_{2}\right)\left(r_{2}+r_{2}\right)
$$

where $D_{1}=k T / 6 \pi \eta r_{1}$ and $\eta$ is the viscosity of the gas.

In terms of volume the kernel can be written as:

$$
K_{\mathrm{B}}(u, v)=\frac{2 k T}{3 \eta}\left(2+\left(\frac{u}{v}\right)^{\frac{1}{3}}+\left(\frac{v}{u}\right)^{\frac{1}{3}}\right)
$$

where $u=4 \pi r_{1}^{3} / 3$, etc.

Since the particle density does not occur, this kernel is independent of the material of which it is composed and depends on the total volume only. We should note, however, that it is based upon the spherical particle approximation. That is when two spherical particles collide they form a new spherical particle whose volume is the sum of the individual volumes. In practice, the aerosols are not spherical and this raises many problems some of which will be discussed later.

3.1.2. Gratitational settling. Another important mechanism for coagulation arises from the differential settling of particles of different masses. The simplest form of this kernel is;

$$
\begin{aligned}
K_{\mathrm{G}}(u, v) & =\pi\left(r_{1}+r_{2}\right)^{2}\left|V_{\mathrm{T}}\left(r_{1}\right)-V_{\mathrm{T}}\left(r_{2}\right)\right| \\
& =\text { collision cross section } \times \text { relative velocity }
\end{aligned}
$$

where $V_{\mathrm{T}}(r)$ is the settling velocity of a particle of radius $r$.

Using Stokes' law leads to:

$$
V_{\mathrm{T}}(r)=\frac{m g}{6 \pi \eta r}
$$

and hence:

$$
K_{\mathrm{G}}(u, v)=\frac{g}{6 \eta}\left(r_{1}+r_{2}\right)^{2}\left|\frac{m_{1}}{r_{1}}-\frac{m_{2}}{r_{2}}\right| .
$$

If the two particles consist of a single species, i.e. are made of the same material, then we can write for the density $\rho_{\mathrm{p}}$ :

$$
\rho_{\mathrm{p}}=\frac{3 m_{1}}{4 \pi r_{1}^{3}}=\frac{3 m_{2}}{4 \pi r_{2}^{3}}
$$

where

$$
K_{\mathrm{G}}(u, v)=\frac{\rho_{\mathrm{\rho}} g}{6 \eta}\left(\frac{3}{4 \pi}\right)^{\frac{1}{3}}\left(v^{\frac{1}{3}}+u^{\frac{1}{3}}\right)^{2}\left|u^{\frac{2}{3}}-v^{\frac{2}{3}}\right| .
$$

This result does not include any interparticle forces induced by the fluid which lead to deviations from the geometric collision cross section.

There are several other types of coagulation mechanism, for example; (1) laminar shear, (2) turbulent shear and (3) turbulent inertial. These turbulent mechanisms are strongly dependent on the temperature and velocity of the gas in the containment structure.

Although analytical expressions exist for each of the coagulation mechanisms mentioned above, they are subject to a number of serious limitations. All are recognized, but few have really been settled in a satisfactory manner.

Four important limitations are:

(1) All particles are spherical before and after a collision.

(2) The fluid effects which cause interparticle forces are incorporated by a multiplicative correction factor known as the collision efficiency. 
(3) Coagulation mechanisms are additive. That is to say,

$$
K_{\text {total }}=K_{\mathrm{B}}+K_{\mathrm{G}}+\cdots
$$

(4) Corrections must be applied when the particles are comparable to a mean free path.

We shall now briefly discuss these problems.

(1) The question of sphericity seems to be a fundamental problem. It is only necessary to look at photomicrographs of aerosols during their aging process to see that they are certainly not spherical. Indeed, in some cases, they are more like long chain-like objects than spheres. This non-sphericity will affect the coagulation in two important ways: It increases the drag and so reduces settling velocities and it increases the effective collision cross section thereby increasing the coagulation rate. The present technique for dealing with non-sphericity is to use equivalent aerodynamic diameters, but this is open to objection since it assumes that as the aerosol ages, its aerodynamic correction factor remains constant. If a particle changes shape significantly during aging, as many do, this will lead to failure of the aerodynamic diameter concept.

(2) The second source of error involved in the coagulation rate arises from the so-called collision efficiency (Pruppacher and Klett, 1978). This is a correction factor which attempts to allow for the interparticle forces arising from the interaction of two particles in a fluid field. These forces may be calculated from the slow viscous flow equations and if the equations of motion of two approaching particles are solved the trajectories of the particles can be obtained. It is then seen that due to these fluid forces, particles which would have collided on a purely geometric basis, neglecting fluid forces, now do not. For example, very small particles will tend to move along a streamline of the larger particle and therefore not collide.

Thus the effect of interparticle forces is to reduce the coagulation rate. A number of semi-empirical correction formulae have been developed by which the classical coagulation rate is multiplied to give the one used in calculations, e.g.

$$
K_{\mathrm{G}}^{*}(u, v)=\varepsilon(u, v) K_{\mathrm{G}}(u, v)
$$

where $K_{\mathrm{G}}$ is the geometrical value used earlier and $\varepsilon$ is the collision efficiency. Generally, the collision efficiency is less than unity, but for nearly equal particles it can exceed unity due to a wake effect. Whilst these semi-empirical collision efficiencies are used frequently in most current aerosol computer codes, they have never been tested under clean experimental conditions. Moreover, they still have the feeling of a 'fudge factor' approach.

There are ways to improve this situation but it would involve some computational effort which might not be justified for frequent commercial use, but at least would give some confidence, or not, in the currently used recipes.

(3) The third source of potential error in the coagulation rate arises from the assumption that the coagulation rates for the individual mechanisms can be added. This really is a gross assumption and other than the case of turbulent and gravitational coagulation, until recently no attempt has been made to combine the processes in a consistent manner.

The gravitational and turbulent calculations were carried out in 1956 (Saffman and Turner, 1956) and it was not until 30 years later that the basic problem of simultaneous coagulation by gravitational motion and Brownian diffusion was solved (Simons et al., 1987). In that case it was discovered that the error incurred in simply adding the Brownian and gravitational kernels amounted to about $27 \%$ in the worst case.

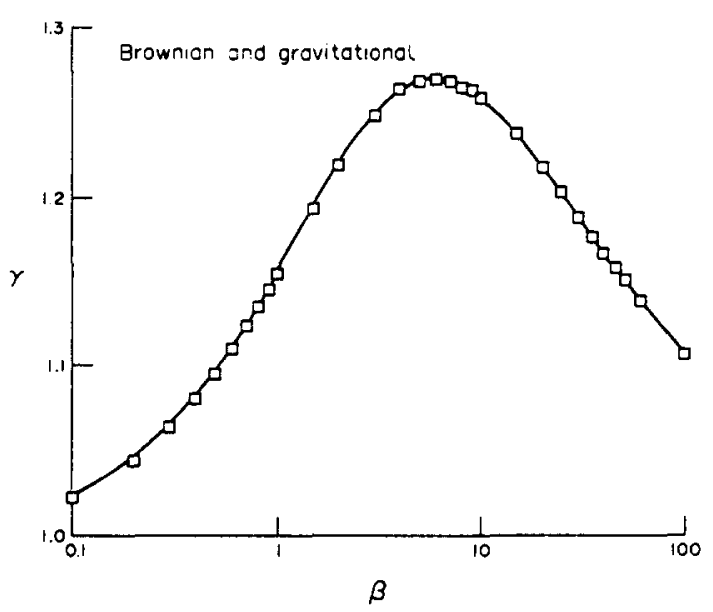

Fig. 3. Ratio of exact Brownian-gravitational coagulation kernel to the sum approximation. $\beta$ is a measure of gravitational to diffusive forces.

Figure 3 shows the ratio of the exact kernel to the sum kernel for a range of values of $\beta$. Where $\beta$ is a measure of the ratio of gravitational to diffusive forces.

These calculations have since been extended to obtain coagulation kernels for simultaneously acting Brownian, turbulent and gravitational coagulation (Williams, 1988). This method, is based upon solutions of the diffusion-advection equation;

$$
\nabla \cdot D(\mathbf{r}) \nabla C(\mathbf{r})-\nabla \cdot[\mathbf{V}(\mathbf{r}) C(\mathbf{r})]=0
$$

where $D$ is the diffusion coefficient and $\mathbf{V}$ is the relative velocity between the particles. The diffusion coefficient and the relative velocity can be obtained by 
separate albeit quite complicated methods (Batchelor, 1982; Pruppacher and Klett, 1978). Thus we can include interparticle forces in the calculation both in the convective and diffusive terms. It has only recently been recognized by nuclear aerosol workers that the Brownian diffusion coefficient is in fact a function of interparticle distance. This leads to a reduction in the rate of coagulation arising from Brownian diffusion. The effect is well-known to colloid scientists.

(4) Finally, there are corrections needed when the particles are comparable to a mean free path in size. These effects are generally allowed for by means of a semi-empirical factor called the Cunningham correction factor and this is one area in which, while the theory is not as rigorous as might be wished, the results do give good agreement with experiment (Friedlander, 1977). It seems, therefore, that any additional effort needed in the kinetic theory regime should be directed towards non-spherical effects and also towards thermo- and diffusiophoresis.

\subsection{Reduction of the balance equation}

Having discussed the various parameters that appear in the aerosol equation, it is now necessary to actually solve it. It seems unlikely that we can handle the equation as it stands, even with only one species. So we will describe the current approximate procedure. The containment vessel is divided into a number of compartments each of which covers a major component or region. There may be as many as 10 of these regions according to the complexity of the geometry involved. Moreover, account must be taken of the inflow and outflow of gas from the compartments which transport the aerosol. If the containment has $N$ compartments it is not difficult to show that by volume averaging over the compartments our single aerosol equation is reduced to a set of $N$ coupled equations of the form (Williams, 1986):

$$
\begin{aligned}
\frac{\partial}{\hat{c} t} & \left(V_{i} n_{i}(v, t)\right)+V_{i} R_{i}(v) n_{i}(v, t) \\
& +\frac{\partial}{\partial v}\left(V_{i} I_{i}(v) n_{i}(v, t)\right)=\sum_{j=1}^{N} \alpha_{j-i}(v, t) V_{j} n_{j}(v, t) \\
& -V_{i} n_{i}(v, t) \sum_{j=1}^{\mathrm{N}} x_{i \rightarrow j}(v, t) \\
& +\frac{1}{2} V_{i} \int_{0}^{v} \mathrm{~d} u K_{i}(v-u, u) n_{i}(u, t) n_{i}(v-u, t) \\
& -V_{i} n_{i}(v, t) \int_{0}^{\infty} \mathrm{d} u K_{i}(u, v) n_{i}(u, t)+V_{i} S_{i}(v, t) \\
i= & 1,2, \ldots \mathrm{N} .
\end{aligned}
$$

$V_{i}$ are the volumes of the compartments. The $x_{i j}$ describe the rate at which particles are transferred from compartment $i$ to compartment $j$. They depend upon the gas dynamics of the problem and on the way in which the compartments are coupled to each other.

The terms $R_{i}(v)$ account for removal processes arising from diffusion to surfaces, and to gravitational settling on the floor. They can also be modified to incorporate such effects as thermophoresis and diffusiophoresis. These processes are particularly difficult to obtain quantitatively from first principles because they require knowledge of the temperatue and concentration gradients across thin boundary layers. They depend therefore on various heat transfer correlations.

Having reached this stage, we now have to obtain numerical solutions of these $\mathrm{N}$ coupled integrodifferential equations. There is a variety of ways to do this. Several computer codes exist, developed in various national laboratories, which carry out numerical integration of the aerosol equations, some are:

(1) MAEROS Sandia National Laboratory (finite difference).

(2) AEROSOLS Commisariat a l'Energie Atomique (finite element).

(3) AEROSIM United Kingdom Atomic Energy Authority (finite difference).

(4) NAUA Kernforschungszentrum Karlsruhe (finite difference).

(5) RAFT Argonne National Laboratory (finite difference).

(6) HAARM Atomics International (moments).

Each of these codes has advantages and disadvantages. Some include certain physical processes and exclude others and some are based upon volume discretization and others on radius discretization. Yet others have been verified to a certain extent experimentally. However, the major disadvantage of most of the codes (the exception is HAARM) is the large amount of computer time needed. This can become exceptionally large if several compartments and aerosol species are considered. Thus there are major economic advantages to be gained by seeking other methods of solution.

It is worthwhile examining various ways of improving the efficiency of aerosol calculations using what might be termed semi-analytical methods, in this case the method of moments. One of the first attempts to solve the aerosol equations was carried out by Cohen and Vaughan (1971). Their idea was to assume an analytical form for the aerosol distribution function containing a few free parameters. By inserting this ansatz into the aerosol equation and taking volume moments, a set of equations equal to the number of free parameters was obtained which could be solved very 
easily. Cohen and Vaughan used as the basis function the log-normal distribution, viz.:

$$
n(v, t)=\frac{N(t)}{(2 \pi \sigma(t))^{\frac{1}{2}}} \frac{1}{v} \exp \left\{-\frac{1}{2 \sigma(t)} \ln ^{2}\left(\frac{v}{\hat{v}(t)}\right)\right\} .
$$

This has three free parameters $N, \hat{v}$, and $\sigma$. Thus we need three moments to be satisfied and this leads to three equations for $N, \hat{v}$ and $\sigma$. In the equivalent numerical method one may require several hundred mesh points to adequately describe the situation and this means solving several hundred equations instead of three. This is the basis of the HAARM code.

Of course, the effectiveness of this method depends on how well the log-normal distribution describes the actual situation. It has been noted during the past few years that the log-normal leads to serious underestimates of the number of particles and the amount of material in suspension after long times. For this reason, the log-normal method and with it the method of moments fell into disrepute. However, the method is so attractive in principle that a program of research was carried out to see whether some other ansatz could be used to greater effect (Williams, 1986). To this end we considered the possibility of using a modified gamma distribution, known in statistics as the Weibull distribution, viz.:

$$
n(v)=\frac{N \beta}{\Gamma\left(\frac{v+1}{\beta}\right)} \frac{1}{\bar{v}}\left(\frac{v}{\bar{v}}\right)^{v} \exp \left[-\left(\frac{v}{\bar{v}}\right)^{\beta}\right] .
$$

There are four free parameters, $N, v, \beta$ and $\bar{v}$. Thus we need four moments to be satisfied. The other condition required of the ansatz is that the double integrals appearing in the coagulation terms be expressible in terms of tabulated or readily calculated functions. For all important coagulation processes, the modified gamma distribution satisfies this requirement.

To illustrate the accuracy of the modified gamma distribution, consider the case of brownian coagulation and gravitational settling in a single chamber. It is convenient when doing this calculation to use a nondimensional time:

$$
\tau=\frac{2 \rho_{\mathrm{p}} g r_{0}^{2} t}{9 \eta H}
$$

where $H=10 \mathrm{~m}, N_{0}=9.15 \times 10^{13} \mathrm{~m}^{-3}, r_{0}=0.34 \mathrm{mic}$ rons, $T=300 \mathrm{~K}, \rho_{\mathrm{p}}=1000 \mathrm{kgm}^{-3}, \quad \eta=1.8 \times 10^{-5}$ $\mathrm{kgm}^{-1} \mathrm{~s}^{-1}$, in terms of these parameters $t=200 \tau(\mathrm{hr})$.

This data corresponds physically to water droplets in air. Figures 4 and 5 show the results for the lognormal, modified gamma and the numerical solution from AEROSIM. It is clear that the log-normal

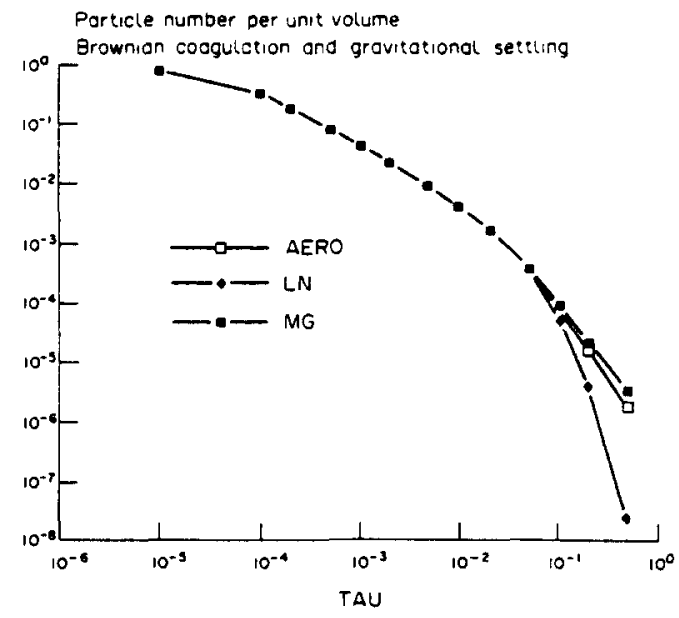

Fig. 4. Particle number per unit volume normalized to the initial value as a function of time. Brownian coagulation and gravitational settling. ( $\mathrm{LN}=\log$-normal, $\mathrm{MG}=$ modified Gamma, AERO $=$ AEROSIM code.)

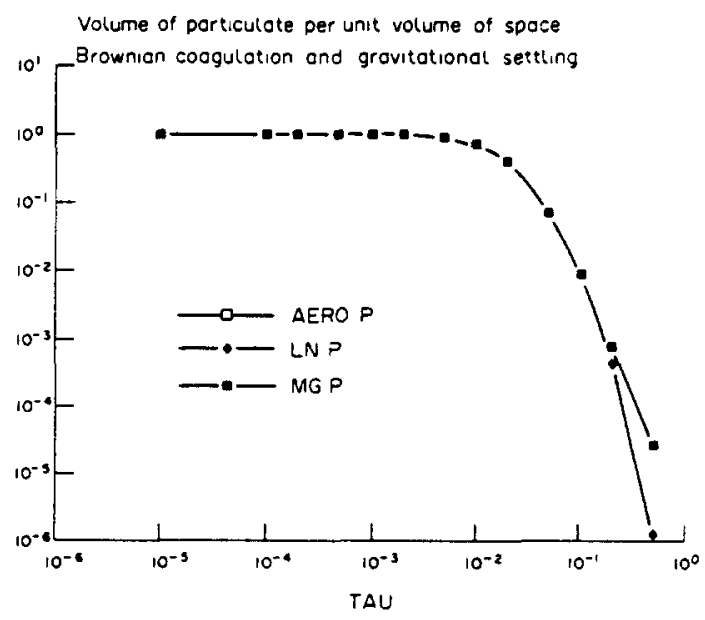

Fig. 5. Volume of particulate matter per unit volume of space normalized to the initial value as a function of time. Legends as for Fig. 4.

distribution can underestimate the AEROSIM result by an order of magnitude. On the other hand, the modified gamma distribution is very close to the AEROSIM values. This is especially noticable at long times after the accident which are generally the important times as far as accidents are concerned.

Further encouraging results using the modified gamma distribution have been obtained for other coagulation and removal mechanisms. For example, Simpson et al. (1988) have modelled an aerosol release 
in a three compartment LWR containment and also a four compartment release in a nuclear submarine. Realistic data has been used and excellent agreement found between the modified gamma distribution and the results from the code AEROSIM, but with a reduction in computing time of about a factor of 10 .

The purpose of these remarks is to emphasize that there is still a role for the analyst in the solution of the aerosol equations; it is not always necessary to use brute force to obtain useful results.

\section{SUMMARY}

In summary then, we can draw the following conclusions:

(1) Severe accident analysis involves complex interactions among many disparate phenomena.

(2) It is impossible to eliminate every degree of uncertainty from the analysis because containment calculations themselves are uncertain and there are limitations arising from the phenomenological knowledge that has to be used.

(3) That part of the calculation due to aerosol behavior can be formulated fairly rigorously but many of the parameters that enter into the formulation are not well known. Some inaccuracy is acceptable and often it is not necessary to obtain extremely accurate values of certain parameters if the figure of engineering interest does not merit it.

(4) Execution times can be dramatically reduced by means of semi-analytic methods but these have to be used with caution since their region of validity is not fully known.

\section{FUTURE WORK}

(1) There are many uncertainties in the general formulation of aerosol behavior. It is therefore impor- tant to carry out a sensitivity analysis, i.e. to vary the parameters and see what effect this has on the particle distribution.

(2) The strong interaction between the aerosol properties and the thermal hydraulics should be investigated more closely.

(3) There is scope for further improvement in the calculation of collision efficiency and shape factor.

(4) The charge state of the aerosol is usually neglected. Whilst, on average, we expect the positive and negative effects to cancel, we ought to be more precise about this. For example, does surface charging affect the deposition rate? How does the radioactive environment of the aerosol affect the charging rate? Time scales of charging rates compared with agglommeration rates are very important here, e.g. if the aerosol charge has not reached equilibrium then the approximation of overall neutrality may not be valid.

(5) Small scale experiments will play a vital role in assessing the system parameters such as, collision efficiency, shape factor, charge state, evaporation and condensation and the chemical reactions that arise.

\section{REFERENCES}

Batchelor G. K. (1982) J. Fluid Mech. 119, 379

Cohen E. R. and Vaughan E. U. (1971) J. Coll. Interface Sci. 35,612 .

Farmer F. R. (1967) Nucl. Saf. 8, 539.

Friedlander S. K. (1977) Smoke, Dust and Haze, WileyInterscience.

Pruppacher H. R. and Klett J. D. (1978) Microphysics of Clouds and Precipitation, D. Reidel.

Simons S., Williams M. M. R, and Cassell J. S. (1987) J. Aerosol Sci. 17, 789.

Simpson D., Williams M. M. R., and Simons S. (1988) Nucl. Sci. Engng, in press.

Williams M. M. R. (1986) Prog. nucl. Energy 17, 1. Williams M. M. R. (1988) J. Phys. D: appl. Phys. 21, 875. Wilson R., et al. (1985) Rev. mod. Phys. 57, S1. 